\title{
Clinical diagnostic value of digestive endoscopic narrow-band imaging in early esophageal cancer
}

\author{
ZHENHUA SU $^{1}$, LIANG WANG ${ }^{2}$, SICHEN WEI $^{1}$, XINLIANG WEI $^{1}$, YU KONG $^{1}$, \\ WEIWEI WANG ${ }^{1}$, RUIXUE GUO ${ }^{1}$ and XIAOMENG SHI ${ }^{1}$ \\ Departments of ${ }^{1}$ Gastroenterology and ${ }^{2}$ Endoscopy Center, Cangzhou Central Hospital, \\ Cangzhou, Hebei 061000, P.R. China
}

Received November 21, 2018; Accepted March 29, 2019

DOI: $10.3892 / \mathrm{ol} .2019 .10278$

\begin{abstract}
Clinical diagnostic value of digestive endoscopic narrow-band imaging in early esophageal cancer (EC) and benign lesions was explored. Retrospective analysis was carried out on the clinical data of 186 patients with early EC and benign lesions diagnosed by the Department of Gastroenterology in Cangzhou Central Hospital from February 2011 to April 2018. Among them, 102 patients examined by Narrow Band Imaging (NBI) were regarded as the research group. Eighty-four patients examined by conventional white light staining endoscopy were regarded as the control group. The lesion boundary definition, image clarity, sensitivity, specificity, positive predictive value, negative predictive value, diagnostic compliance rate, detection rate of lesions and adverse reactions were compared between the groups after examination. The results showed that the lesion boundary definition in the research group was higher than that in the control group $(\mathrm{P}<0.05)$. The 4-points of image clarity in the research group was higher than that in the control group $(\mathrm{P}<0.05)$. The sensitivity, specificity, positive predictive value, negative predictive value, and diagnostic compliance rate of the early EC and benign lesions in the research group were higher than those in the control group $(\mathrm{P}<0.05)$. The detection rate of the upper, middle and lower segments of lesions in the research group was higher than that in the control group. In conclusion, NBI has higher sensitivity, specificity, positive predictive value, negative predictive value, and diagnostic compliance rate for the diagnosis of early EC and benign lesions. Also it has more accuracy in the detection of lesions and fewer adverse reactions, the screening of early EC and benign lesions is effective, and worth promoting clinically.
\end{abstract}

Correspondence to: Dr Sichen Wei, Department of Gastroenterology, Cangzhou Central Hospital, 16 Xinhua West Road, Cangzhou, Hebei 061000, P.R. China

E-mail:w2u6en@163.com

Key words: early esophageal cancer, narrow-band imaging technique, conventional white light staining endoscopy, diagnostic value

\section{Introduction}

Esophageal cancer (EC) is a malignant tumor on the esophageal mucosa at the location of pathogenesis. It is one of the most common digestive tract tumors, very common in most countries and regions around the world, with a high mortality rate (1). The main clinical symptoms of EC are pain, shortness of breath, cough, weight loss, and sometimes patients may experience nausea, diarrhea and satiety (2). The incidence rates of EC has increased over the years. It is a type of malignant tumor with fast growth and high incidence. Also the five-year survival rate is only $\sim 13 \%$. It is predicted that the incidence of EC adenocarcinoma will increase 40-50\% by 2026 (3). EC is the most common digestive tract tumor, and has the 7th highest mortality rate among all cancers in the world (4). The main reason for the high mortality rate is that most patients are already in the advanced stage at the time of diagnosis, missing the opportunity for surgery (5). In order to improve the prognosis of patients with EC, chemotherapy is often used for the treatment of cancer to achieve a reduction of the diameter of the tumor. However, chemotherapy with concurrent radiotherapy will increase the level of drug toxicity and other toxic side effects. This can even lead to delayed surgery time of EC patients who are not sensitive to chemotherapy and concurrent radiotherapy, which further causes delayed treatment (6). Studies have shown that early EC has no lymph node metastasis and no vascular infiltration, the five-year survival rate of patients is generally high, $60-70 \%$, with a good prognosis (7). This shows that early detection and early diagnosis of EC can improve the survival rate of EC patients and increase the effectiveness of treatment, it has an important significance in EC patients.

In order to diagnose EC as early as possible, endoscopic biopsy is often used in high-risk patients for diagnosis. However, this method is prone to sampling errors and the accuracy of the method is also affected by the tester at the same time (8). In recent years, the diagnosis of EC tissue tumor markers related to gene and cell abnormalities has also attracted the attention of the many medical scientists. However, due to the limitations of current medical technology, changes in EC tumor markers are difficult to detect in the early stages of cancers, and are of little significance in the diagnosis of early EC (9). Identification of an early EC diagnostic method 
with higher accuracy and more convenience has become a hot topic in EC research.

Narrow Band Imaging (NBI) is an advanced endoscopic technique. Compared to conventional white endoscopy, NBI technology uses selective filtering of light, focuses light on the mucous membrane surface and provides reliable sensing performance for tissue biopsy (10). NBI is formed by the three narrow-band light waves of green and blue with wavelengths of 540 and $415 \mathrm{~nm}$. Visually enhancing the affected area with light sources of different wavelengths, is more convenient in the application of qualitative and targeted biopsy on the lesion (11). NBI, which has been widely used, is easy to operate, with no dye agent required under the endoscope, it has a similar imaging effect to white light stained endoscope. Compared to white light staining endoscopy, it has a better NBI detection, and the false positive detection rate is similar to white light staining endoscopy (12).

This study examined the status of early EC and benign lesions by using NBI, the value of NBI in the diagnosis of early $\mathrm{EC}$ and benign lesions were analyzed, to provide evidence for clinics.

\section{Materials and methods}

General information. Retrospective analysis was carried out on the clinical data of 186 patients with early EC and benign lesions diagnosed by the Department of Gastroenterology in Cangzhou Central Hospital (Cangzhou, China) from February 2011 to April 2018. Among them, 102 patients examined using NBI were regarded as the research group, including 65 cases of males, 37 cases of females, with an average age of $50.23 \pm 15.93$ years. Eighty-four patients examined by conventional white light staining endoscopy were regarded as the control group, including 54 cases of males, 30 cases of females, with an average age of $52.31 \pm 16.73$ years. All patients voluntarily accepted the necessary examinations, and all of them had signed the informed consent form. The study was approved by the Ethics Committee of Cangzhou Central Hospital.

\section{Inclusion and exclusion criteria}

Inclusion criteria: Age $\geq 18$ years; the red and gray color appeared in the area of esophageal and hypopharyngeal mucosa; the surface of the esophagus and hypopharynx mucosa is not smooth, matte, or accompanied by erosion; the mucosa of the esophagus and hypopharynx was thickened, and the structure of the vascular network was blurred or disappeared; and patients with complete clinical medical records.

Exclusion criteria: Suffering from acute pharyngeal infection; patients with iodine allergy or have had a history of hyperthyroidism; patients cannot perform painless gastroscope operation for special reasons; and the esophagus and hypopharyngeal mucosa were still unclear after washing.

Experimental equipment. Gastroscope host (model: CV-260SL); HD electronic magnifying endoscope (model: GIF-H260); light source (model: CLV-260) (all from Olympus Japan Ltd., Tokyo, Japan); Rugo's iodine solution (code: G1069; Beijing Suo Laibao Technology Co., Ltd., Beijing, China); simethicone (National Pharmacy: H20170002; Nanjing Hanstone Pharmaceutical Co., Ltd.,
Nanjing, China); 2\% lidocaine gel (National Pharmaceutical code: H11022396; China Resources Zizhu Pharmaceutical Co., Ltd., Beijing, China); butyl bromide scopolamine injection (national medicine quasi-font: H32023810; Jiangsu Ange Pharmaceutical Co., Ltd., Jiangsu, China); endoscopic mist spray tube (model: AF-2416PB; Olympus Japan Ltd.) were used in the present study. Dual system endoscope was used in this study, and regular white endoscopy can be performed. The system was switched to NBI mode for detection where necessary.

\section{Method of detection}

Preparation before detection. Before endoscopy, the patient was examined for blood routine, liver and kidney function, chest CT, and coagulation function, to ensure that endoscopic contraindications were excluded. On the day of endoscopy, patients were inhibited for drinking water and fasted for $10 \mathrm{~h}$. Patients' blood pressure and electrocardiogram were checked $60 \mathrm{~min}$ before the detection. If there was no abnormality, patients took $30 \mathrm{ml}$ of defoamer simethicone and 1,000 $\mathrm{ml}$ of distilled water $30 \mathrm{~min}$ before the detection in order to remove mucus and air bubbles from the detection area, improve the vision and increase the disease detection rate. Five minutes before the examination, patients were given $2 \%$ of lidocaine gel for local pharyngeal anesthesia, the head was placed in the back position and the drug kept in full contact with the pharynx, an intravenous channel was established after anesthesia, and intravenous injection of butyl bromide was used to reduce gastrointestinal motility.

Detection process. The patient lay on the left side, leaning forward and bending the legs inward. The research group was examined by ordinary white light endoscopy. Then the presence of lesions was carefully observed in the esophagus to the duodenum, the lesions were rinsed, switching to NBI mode for detection. The clarity was judged by observing the suspected lesion area, the lesion location, size, edge, morphology, extent and mucosal characteristics were recorded. Also the control group was examined by conventional white light staining, the endoscopic mist spray tube was used to evenly spray the Rugo's iodine solution to the detection area. The clarity was judged by making the coloration contrast of the detection area clearer, and the lesion location, size, edge, shape, extent and mucosal characteristics were recorded. After the above operation was completed, the two groups received two biopsies from the most suspicious lesions under endoscopy, which were sent for condition detection of the lesions.

\section{Experimental evaluation criteria}

Assessment of the clarity of lesion boundary. Clear: The extent of the lesion is very clear, and the boundary between the lesion and the normal tissue is clearly defined. Blurry: The lesion range is unclear and the lesion boundary is sticky with normal tissue. Pathological clarity of the lesion $=$ number of people with cleared clarity/total number of people.

Evaluation of image clarity. 1-point, blurred image; 2-points, faintly visible image; 3-points, visible image; 4-points, clearly visible image.

Diagnostic criteria. The research group used the NBI magnifying lens to observe the intrappillary capillary loop (IPCL) on the lesion area. The IPCL changes are classified 
Table I. Comparison of general baseline data between the research group and the control group [n (\%)] (mean \pm SD).

\begin{tabular}{|c|c|c|c|c|}
\hline Types & $\begin{array}{c}\text { Research } \\
\text { group }(n=102)\end{array}$ & $\begin{array}{c}\text { Control } \\
\text { group }(n=84)\end{array}$ & $\chi^{2} / \mathrm{T}$ & P-value \\
\hline Age (years) & $50.23 \pm 15.93$ & $52.31 \pm 16.73$ & 0.866 & 0.387 \\
\hline Sex & & & 0.065 & 0.879 \\
\hline Male & $65(63.73)$ & $52(61.90)$ & & \\
\hline Female & $37(36.27)$ & $32(38.10)$ & & \\
\hline $\mathrm{BMI}\left(\mathrm{kg} / \mathrm{m}^{2}\right)$ & & & 0.060 & 0.850 \\
\hline$<24$ & $84(82.35)$ & $68(80.95)$ & & \\
\hline$\geq 24$ & $18(17.65)$ & $16(19.05)$ & & \\
\hline Heart rate (times/min) & & & 0.941 & 0.411 \\
\hline$<60$ & $13(12.75)$ & $15(17.86)$ & & \\
\hline$\geq 60$ & $89(87.25)$ & $69(82.14)$ & & \\
\hline Smoking status & & & 0.125 & 0.762 \\
\hline Smoking & $63(61.76)$ & $54(64.29)$ & & \\
\hline Non-smoking & $39(38.24)$ & $30(35.71)$ & & \\
\hline Fasting blood sugar (mmol/l) & $4.81 \pm 0.72$ & $4.68 \pm 0.58$ & 1.336 & 0.183 \\
\hline $\mathrm{Hb}(\mathrm{g} / \mathrm{l})$ & $121.48 \pm 17.27$ & $125.93 \pm 14.71$ & 1.868 & 0.063 \\
\hline $\operatorname{RBC}\left(x 10^{12} / 1\right)$ & $4.48 \pm 0.46$ & $4.53 \pm 0.41$ & 0.775 & 0.440 \\
\hline $\operatorname{PLT}\left(\times 10^{9} / 1\right)$ & $224.82 \pm 56.16$ & $226.35 \pm 54.26$ & 0.188 & 0.851 \\
\hline Alcoholism & & & 0.033 & 0.883 \\
\hline Alcoholic & $56(54.90)$ & $45(53.57)$ & & \\
\hline Non-alcoholic & $46(45.10)$ & $39(46.43)$ & & \\
\hline
\end{tabular}

into 4 types according to Swangsri et al (13) standard: Type 1, the capillaries in the papillary are in the shape of regularly arranged thin ring. Type 2 , the capillaries in the papillary are in the shape of regularly arranged thin ring, however, it can be seen that the pipe diameter is enlarged or extended. Type 3, destroyed shape of capillaries in the papillary, uneven diameter size, irregularly arranged and even appeared in a shape of a bended snake. Type 4, destroyed capillaries in the papillary, blood vascular appeared in an overlapped distribution, new tumor blood vessels appeared and have irregular branches. Type 1 is considered as normal esophageal mucosa; type 2 is considered to be a benign lesion of the esophagus; type 3 and 4 is considered as EC; types 1 and 2 are classified as negative pathology and type 3 and 4 are classified as positive pathology.

After the control group was stained with Rugo's iodine solution, the stain appeared brown, light brown or no color. Brown is an indication of normal esophageal mucosa, light brown indicates benign lesion of the esophagus and no color indicates EC (14). Brown and light brown were considered as a negative case, and no color was considered as a positive case.

Assessment of diagnostic results. Extract analysis of four tables of diagnostic experiments, sensitivity calculation, specificity levels, positive diagnostic and negative predictive value and diagnostic compliance rate.

Nursing care after detection. After the detection, patients were sent to the recovery room. Monitoring of the patient's vital signs was continued until the patient had vomiting reflexes and pharyngeal sensation recovery. Adverse reactions such as vomiting and gastrointestinal bleeding in the patient were observed and recorded within $1 \mathrm{~h}$ after the examination. Patients were left alone when they had the ability to swallow.

Statistical analysis. Statistical analysis was performed using SPSS 17.0 (Beijing Sitron Weida Information Technology Co., Ltd., Beijing, China). Countable data was expressed as a percentage, and measurement data were expressed as mean \pm standard deviation (means $\pm \mathrm{SD}$ ). The Chi-square test was used to compare countable data between groups and t-test was used to compare the measurement data between groups. The rank sum test was used to analyze the grade data, expressed as $\mathrm{Z}$. $\mathrm{P}<0.05$, was considered as statistically significant.

\section{Results}

Comparison of general baseline data. The age, sex, body mass index, heart rate, smoking status, fasting blood glucose level, hemoglobin $(\mathrm{Hb})$ value, red blood cell (RBC) value, platelet (PLT) value, and alcoholism were compared between the research group and the control group. The difference between two groups was not statistically significant $(\mathrm{P}>0.05)$ (Table I).

Comparison of clarity between the research group and the control group

Comparison of the boundary definition between the research group and the control group. According to the result of images in both groups under NBI endoscopy and ordinary white light staining endoscopy, in the research group, there 


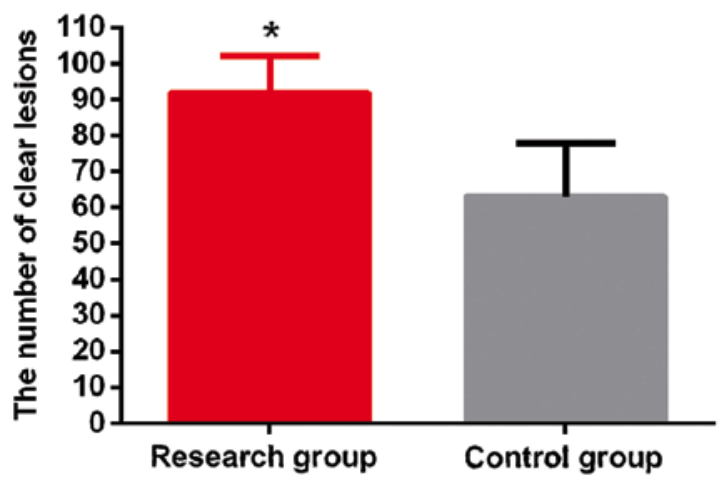

Figure 1. Comparison of lesion boundary between the research group and the control group. The lesion boundary definition in the research group was $90.20 \%$ and was $75.0 \%$ in the control group. The lesion boundary definition in the research group was higher than that in the control group $(\mathrm{t}=7.659$, $\mathrm{P}<0.01) .{ }^{*} \mathrm{P}<0.05$ was considered to indicate a statistically significant difference compared with the control group.

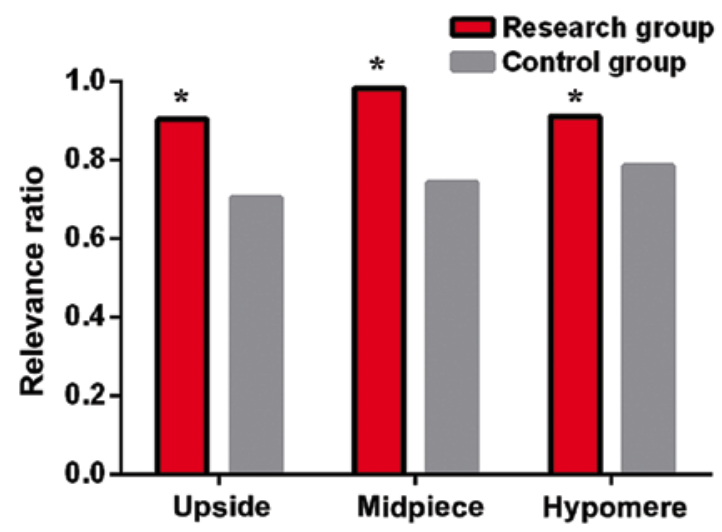

Figure 2. Comparison of the detection of lesions between the research group and the control group. The NBI test results in the research group were $90.48 \%$ in the upper segment, $89.36 \%$ in the middle segment, and $91.18 \%$ in the lower segment. The results of white light staining endoscopy in the control group were $70.59 \%$ in the upper segment, $74.36 \%$ in the middle segment, and $78.57 \%$ in the lower segment. The detection rate in the area of upper, middle and lower lesions of the research group was higher than that in the control group $\left({ }^{*} \mathrm{P}<0.05\right)$.

were 92 cases of cleared lesions and 10 blurred cases and the boundary definition of the lesion was $90.20 \%$. In the control group, there were 63 cases of clear lesions, 21 blurred cases and the boundary definition of the lesion was $75.00 \%$. The lesion boundary definition in the research group was higher than that in the control group, and the difference between the two groups was statistically significant $(\mathrm{t}=7.659, \mathrm{P}<0.01)$ (Fig. 1).

Image clarity between the research group and the control group. According to the results of imaging in both groups under NBI endoscopy and ordinary white light staining endoscopy, the image clarity in the research group was classified into 4 points, 3 points, 2 points and 1 point, each had 74 cases, 20 cases, 6 cases and 2 cases, respectively. The image clarity in the control group was classified as 4 points, 3 points, 2 points and 1 point, each had 36 cases, 27 cases, 13 cases and 8 cases, respectively. The image clarity in the research group was higher than that in the control group, and the difference was statistically significant $(\mathrm{t}=16.806, \mathrm{P}<0.01)$. The 3 points, 2 points, and 1 point of image clarity in the research group

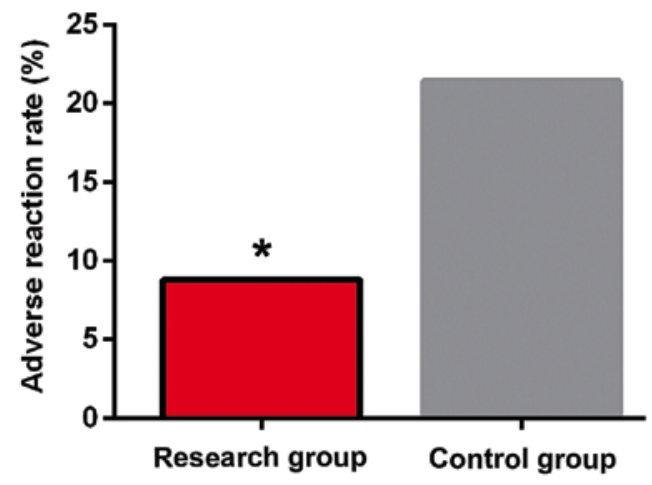

Figure 3. Comparison of adverse reactions between the research group and the control group. The adverse reaction rates in the research group was $8.82 \%$ and was $21.43 \%$ in the control group. The adverse reaction rates in the research group was better than that in the control group. The difference between two groups was statistically significant $(\mathrm{t}=5.898, \mathrm{P}=0.021)$. ${ }^{*} \mathrm{P}<0.05$ was considered to indicate a statistically significant difference compared to the control group.

were lower than those in the control group. By contrast the 4 points was higher than that of the control group. The difference was statistically significant $(\mathrm{P}<0.05)$ (Table II).

Detection of lesions between the research group and the control group. According to the results of histopathological examination, in the study group, there were 21 cases of lesions in the upper segment, 47 cases in the middle segment and 34 cases in the lower segment. In the NBI test results of the research group, there were 19 cases in the upper segment and the detection rate was $90.48 \%, 43$ cases in the middle segment with a detection rate of $91.49 \%, 31$ cases in the lower segment with a detection rate of 91.18. In the control group, there were 17 cases of lesions in the upper segment, 39 cases in the middle segment and 28 cases in the lower segment. In the results of white light staining endoscopy of the control group, there were 12 cases in the upper segment with a detection rate of $70.59 \%, 29$ cases in the middle segment with a detection rate of $74.36 \%, 22$ cases in the lower segment with a detection rate of $78.57 \%$. The detection rate of the upper, middle and lower lesions in the research group was higher than that in the control group (Fig. 2).

Detection of early EC and precancerous lesions between the research group and the control group. According to the results of the detection report between the groups, the sensitivity, specificity, positive predictive value, negative predictive value, and diagnostic compliance rate in the research group were higher than those in the control group, and the difference was statistically significant $(\mathrm{P}<0.05)$ (Table III).

Incidence of adverse reactions after the detection between the research group and the control group. According to the observations within $1 \mathrm{~h}$ between two groups after the detection, in the research group, there were 9 cases of adverse reactions such as vomiting and gastrointestinal bleeding, with an adverse reaction rate of $8.82 \%$. There were 18 cases in the control group, with an adverse reaction rate of $21.43 \%$. The incidence of adverse reactions in the research group was better than that in the control group. The difference between the groups was statistically significant $(\mathrm{t}=5.898, \mathrm{P}=0.021)$ (Fig. 3). 
Table II. Comparison of the image clarity between the research group and the control group.

\begin{tabular}{lcccccr}
\hline Types & 1 point & 2 points & 3 points & 4 points & Z & P-value \\
\hline The research group $(\mathrm{n}=102)$ & $2(1.96)$ & $6(5.88)$ & $20(19.61)$ & $74(72.55)$ & -4.303 & $<0.001$ \\
The control group $(\mathrm{n}=84)$ & $8(9.52)$ & $13(15.48)$ & $27(32.14)$ & $36(42.86)$ & & \\
\hline
\end{tabular}

Table III. Comparison of detection of early EC and precancerous lesions between the research group and the control group [n (\%)].

\begin{tabular}{|c|c|c|c|c|c|c|c|}
\hline & \multirow{2}{*}{\multicolumn{2}{|c|}{ Pathological diagnosis }} & \multicolumn{5}{|c|}{ Diagnostic result (\%) } \\
\hline & & & \multirow[b]{2}{*}{ Sensitivity } & \multirow[b]{2}{*}{ Specificity } & \multirow{2}{*}{$\begin{array}{l}\text { Positive } \\
\text { predictive } \\
\text { value }\end{array}$} & \multirow{2}{*}{$\begin{array}{l}\text { Negative } \\
\text { predictive } \\
\text { value }\end{array}$} & \multirow{2}{*}{$\begin{array}{c}\text { Diagnostic } \\
\text { compliance rate }\end{array}$} \\
\hline & Positive & Negative & & & & & \\
\hline The research group $(n=102)$ & & & 91.57 & 84.21 & 96.20 & 69.57 & 90.20 \\
\hline Positive & 76 & 3 & & & & & \\
\hline Negative & 7 & 16 & & & & & \\
\hline The control group $(n=84)$ & & & 69.23 & 26.32 & 76.27 & 20.00 & 59.52 \\
\hline Positive & 45 & 14 & & & & & \\
\hline Negative & 20 & 5 & & & & & \\
\hline$\chi^{2}$ test & & & 12.193 & 12.880 & 12.422 & 11.959 & 23.996 \\
\hline $\mathrm{P}$-value & & & $<0.01$ & $<0.01$ & $<0.01$ & $<0.01$ & $<0.01$ \\
\hline
\end{tabular}

\section{Discussion}

EC is a very common malignant tumor with high incidence rate, the pathogenesis is currently unclear, however, it has been shown to be associated with genetic changes, such as keratinocyte 7 and keratinocyte 20 (15). Studies have shown that due to the complicated lymphatic system of esophagus, transverse longitudinal connection and complicated pattern of lymph node metastasis, it has become one of the most important factors affecting the prognosis of EC. Particularly, long-term prognosis is affected by the number of invaded lymph nodes (16). EC has a slow development, with many steps and has been divided into multiple stages. Early EC occurs only in the esophageal mucosa or in the superficial layer of the esophagus. It can be cured with endoscopic treatment, and the curative effect is equivalent to the surgery effect. Also patients have less pain, less trauma and faster recovery rate, according to statistics, the five-year survival rate of patients is equivalent to surgery, the diagnosis of early EC has a great significance for the treatment of EC (17).

White light staining endoscopy is one of the commonly used diagnostic methods for EC. However, the microstructure of esophageal mucosa in this method is poorly displayed, also the early EC and esophageal proximal lesions are often missed, EC patients are easily misdiagnosed as benign esophageal ulcers and the miss diagnosis rate is as high as $7.866 \%$ (18). With the development of endoscopy, Olympus Japan Ltd. has developed a new type of magnifying endoscope system: NBI, a very effective non-invasive diagnostic tool with high value for the diagnosis of early cancer (19). The NBI endoscope system uses 415 and $540 \mathrm{~nm}$ narrow band illumination through the
NBI filter, no requirement for separate staining, easy to operate and has a high accuracy in the diagnosis of EC (20).

The present study found that the lesion boundary definition of NBI endoscopy was higher than that of the ordinary white staining endoscopy $(\mathrm{P}<0.05)$. The image clarity of NBI endoscopy was higher than that of the ordinary white staining endoscopy $(\mathrm{P}<0.05)$. According to the results of Gono et al $(21)$, compared with ordinary white stained endoscopy; the NBI endoscopic EC patients have clearer capillaries in the epithelial and mucosal layers. This is similar to our research results, so it indicates that the NBI endoscopy has a higher clarity, which is conducive to accurate judgment of the lesion. This study also found that the sensitivity, specificity, positive predictive value, negative predictive value and diagnostic coincidence rate of NBI endoscopy in the diagnosis of early EC and benign lesions were higher than those of the ordinary white light staining endoscopy $(\mathrm{P}<0.05)$. The detection rates of the upper, middle and lower segments of NBI endoscopic lesions were higher than those of the white light stained endoscopy. Ide et al (22), reported similar results to the present study, and considered that NBI technology has higher sensitivity and negative predictive value for early squamous cell EC patients. Compared with conventional white light staining endoscope, the differences were comparable, the results showed that NBI has a higher value in the diagnosis of EC. The occurrence of adverse reactions were further compared after examination of the patients, the result showed that the incidence of adverse reactions after NBI endoscopy was lower than the conventional white light staining $(\mathrm{P}<0.05)$. We believe that the high adverse reaction rates after conventional white light staining endoscopy may be related to the Rugo's iodine solution used in the examination. 
From the study of Matuszczyk et al (23), it was found that a man was induced by iodine, and caused hyperthyroidism, arrhythmia and angina pectoris. This indicates that iodine has a certain negative impact on the human body.

Overall, the NBI endoscopes are clearer compared with ordinary stained endoscopes, it has a higher levels of sensitivity, specificity, positive predictive value, negative predictive value and diagnostic coincidence rate for early diagnosis of $\mathrm{EC}$ and benign lesions. Also it has a more accurate detection rate of lesions and less occurrence of adverse reactions. It can effectively detect and screen early EC and benign lesions, it is worth promoting clinically.

\section{Acknowledgements}

Not applicable.

\section{Funding}

This study was supported by Science and Technology Project of Cangzhou City, Hebei Province (no. 151302139).

\section{Availability of data and materials}

The datasets used and/or analyzed during the present study are available from the corresponding author on reasonable request.

\section{Authors' contributions}

ZS, LW and SW were responsible for evaluation of image clarity. ZS and XW collected and analyzed general data of patients. YK and WW worked on judgment of the clarity of lesion boundary. RG and XS helped with statistical analysis. The final version was read and adopted by all the authors.

\section{Ethics approval and consent to participate}

The study was approved by the Ethics Committee of Cangzhou Central Hospital (Cangzhou, China). Signed informed consents were obtained from the patients or the guardians.

\section{Patient consent for publication}

Not applicable.

\section{Competing interests}

The authors declare that they have no competing interests.

\section{References}

1. Jain S and Dhingra S: Pathology of esophageal cancer and Barrett's esophagus. Ann Cardiothorac Surg 6: 99-109, 2017.

2. Ginex P, Thom B, Jingeleski M, Vincent A, Plourde G, Rizk N, Rusch VW and Bains M: Patterns of symptoms following surgery for esophageal cancer. Oncol Nurs Forum 40: E101-E107, 2013.

3. Otterstatter MC, Brierley JD, De P, Ellison LF, Macintyre M, Marrett LD, Semenciw R and Weir HK: Esophageal cancer in Canada: Trends according to morphology and anatomical location. Can J Gastroenterol 26: 723-727, 2012.

4. Spallone A and Izzo C: Esophageal cancer presenting as a brain metastasis: A case report. Oncol Lett 6: 722-724, 2013.
5. Wu SX and Wang LH: Current status and perspectives of radiotherapy for esophageal cancer. Zhonghua Zhong Liu Za Zhi 38: 650-654, 2016 (In Chinese).

6. Huang FL and Yu SJ: Esophageal cancer: Risk factors, genetic association, and treatment. Asian J Surg 41: 210-215, 2018.

7. Wang WP, Yang YS, He SL and Chen LQ: Discussion of N staging category of the eighth edition of The AJCC Esophageal Cancer Staging System. Zhonghua Wai Ke Za Zhi 55: 894-897, 2017 (In Chinese).

8. Becker V, Bobardt J, Ott R, Rösch T and Meining A: Long-term follow-up in patients with indeterminate Barrett esophagus. Digestion 88: 161-164, 2013.

9. Shah AK, Saunders NA, Barbour AP and Hill MM: Early diagnostic biomarkers for esophageal adenocarcinoma - the current state of play. Cancer Epidemiol Biomarkers Prev 22: 1185-1209, 2013.

10. Machida H, Sano Y, Hamamoto Y, Muto M, Kozu T, Tajiri H and Yoshida S: Narrow-band imaging in the diagnosis of colorectal mucosal lesions: A pilot study. Endoscopy 36: 1094-1098, 2004.

11. Ladabaum U, Fioritto A, Mitani A, Desai M, Kim JP, Rex DK, Imperiale $\mathrm{T}$ and Gunaratnam N: Real-time optical biopsy of colon polyps with narrow band imaging in community practice does not yet meet key thresholds for clinical decisions. Gastroenterology 144: 81-91, 2013.

12. Chen G, Wang B, Li H, Ma X, Shi T and Zhang X: Applying narrow-band imaging in complement with white-light imaging cystoscopy in the detection of urothelial carcinoma of the bladder. Urol Oncol 31: 475-479, 2013.

13. Swangsri J, Nakajima Y, Kawada K, Tokairin Y, Suzuki T, Miyawaki Y, Hoshino A, Okada T, Ota S, Ryotokuji T, et al: Changes in the microvascular structure of mucosal squamous cell carcinoma of the esophagus and their significance in tumor progression. J Med Dent Sci 60: 83-91, 2014.

14. Dawsey SM, Fleischer DE, Wang GQ, Zhou B, Kidwell JA, Lu N, Lewin KJ, Roth MJ, Tio TL and Taylor PR: Mucosal iodine staining improves endoscopic visualization of squamous dysplasia and squamous cell carcinoma of the esophagus in Linxian, China. Cancer 83: 220-231, 1998.

15. Moreno Racionero F, de Andres Asenjo B, Bedate Nuñez M, Legido Moran P, Ortega Loubon C, Rabadán Jimenez J and Beltran de Heredía Y Rentería J: Unusual relationship between skin lesions and esophageal cancer: A case report and review of literature. Z Gastroenterol 53: 115-119, 2015.

16. Cho JW, Choi SC, Jang JY, Shin SK, Choi KD, Lee JH, Kim SG, Sung JK, Jeon SW, Choi IJ, et al; Korean ESD Study Group: Lymph node metastases in esophageal carcinoma: An endoscopist's view. Clin Endosc 47: 523-529, 2014.

17. Yu X and Wang G: Endoscopic treatment of early esophageal cancer. Zhonghua Wei Chang Wai Ke Za Zhi 18: 860-863, 2015 (In Chinese).

18. Chadwick G, Groene O, Hoare J, Hardwick RH, Riley S, Crosby TD, Hanna GB and Cromwell DA: A population-based, retrospective, cohort study of esophageal cancer missed at endoscopy. Endoscopy 46: 553-560, 2014.

19. Shibahara T, Yamamoto N, Yakushiji T, Nomura T, Sekine R, Muramatsu $\mathrm{K}$ and Ohata $\mathrm{H}$ : Narrow-band imaging system with magnifying endoscopy for early oral cancer. Bull Tokyo Dent Coll 55: 87-94, 2014.

20. Muto M: Endoscopic diagnostic strategy of superficial esophageal squamous cell carcinoma. Dig Endosc 25 (Suppl 1): 1-6, 2013.

21. Gono K, Obi T, Yamaguchi M, Ohyama N, Machida H, Sano Y, Yoshida S, Hamamoto Y and Endo T: Appearance of enhanced tissue features in narrow-band endoscopic imaging. J Biomed Opt 9: 568-577, 2004.

22. Ide E, Carneiro FO, Frazão MS, Chaves DM, Sallum RA, de Moura EG, Sakai P, Cecconello I and Maluf-Filho F: Endoscopic detection of early esophageal squamous cell carcinoma in patients with achalasia: Narrow-band imaging versus Lugol's staining. J Oncol 2013: 736756, 2013.

23. Matuszczyk A, Hahn S, Böse D, Eggebrecht H, Schmermund A, Quadbeck B, Wieneke H, Petersenn S, Janssen OE and Mann K: Gadolinium as an alternative contrast agent during cardiac catheterization in patient with iodine-induced hyperthyroidism. Exp Clin Endocrinol Diabetes 114: 336-338, 2006. 